\title{
Mean-Field Approximation of a Microscopic Population Model for Austria
}

\author{
Martin Bicher ${ }^{1 *}$, Niki Popper ${ }^{2}$ \\ ${ }^{1}$ Institute of Analysis and Scientific Computing, TU Wien, Wiedner Hauptstraße 8-10, \\ 1040 Vienna, Austria; * martin.bicher@tuwien.ac.at \\ ${ }^{2}$ dwh Simulation Services, dwh GmbH, Neustiftgasse 57-59, 1070 Vienna, Austria
}

SNE 28(3), 2018, 117-119 DOI: 10.11128/sne.28.sn.10432

Received: Sept. 15, 2016 (Selected EUROSIM Congress 2016 Postconf. Publ.), Revised July 30, Accepted: August 25, 2018

SNE - Simulation Notes Europe, ARGESIM Publisher Vienna, ISSN Print 2305-9974, Online 2306-0271, www.sne-journal.org

Abstract. Verification and validation of large agent bases models is a complicated process - to check for full functionality, the simulation has to be executed various times, which takes both time and computational resources. In this discussion-paper we present an approach that could generally improve this process, applied on an agent-based population model for Austria. A socalled mean-field model in this case a partial differential equation (PDE) is used for this aim. Execution of the PDE simulation only takes a very short time, hence the meanfield model can provide a fast prospect on results, behaviour and sensitivity of the agent-based model used.

\section{Introduction}

During the last decades a great number of population models have been developed all over the world (e.g. $[1,2,3]))$, which, in combination with economic and/or health models, can be used for supporting internal political decisions. Most of them are microscopic simulation models wherein the population is no longer simulated as a whole [4], but as a sum of individuals.

This type of population modelling is advantageous due to its flexibility with respect to model-extensions and hence plays an important role in the Comet founded healthcare project DEXHELPP. In the course of this project an agent based population model for Austria was developed and implemented [5] in order to create a solid foundation for generating decision-support models for Austria's health care system.

\section{An Agent-Based Population Model for Austria}

The basic rules for the agent-based population model are very intuitive. Each agent is assigned

- age (equivalent to a certain birth-date), and

- sex.

The model is simulated with equidistant time-steps of arbitrary length $d t$. With a certain age and time-steplength dependent probabilities each agent might die, emigrate or, in the case of female agents, reproduce. Additionally a certain number of immigrants is added at each step of the model. Thus the model requires the parameters

- initial population size [\#1],

- initial population distribution with respect to age and sex [\#2],

- immigration age/sex distribution for each simulated time-step [\#3],

- death [\#4], emigration [\#5] and reproduction [\#6] probabilities with respect to age, sex and time-step, and finally

- natural percentage for a male/female offspring [\#7].

These parameters were successfully worked out of requested data from the Austrian Bureau of Statistics [6]. The model was implemented in Python 3.

The simulation executed by CPython 3.4 is very slow. As a single simulation with 8 million agents and a timespan of 50 years with daily time steps takes about 10 hours, the verification and validation process turned out to be challenging. To support that process meanfield analysis was used. 


\section{Mean-Field Analysis}

\subsection{General Concept}

The so called mean-field perhaps poses the most applicable basis to analyse a microscopic model on the macroscopic level. Let $a_{i}(t), i=1 \ldots N$ denote the abstract state of agent $i$ at time $t$ then the mean-field $O(x, t)=\sum_{i=1}^{N} \mathbb{1}_{x}\left(a_{i}(t)\right)$ simply counts all agents in a certain state $x$ at a given time $t$.

In terms of the defined population model the meanfield corresponds to the aggregated numbers: "total population with age $a$ and sex $s$ at time t". As age is a continuous variable and the model is stochastic, the mean-field poses for a density function of a huge stochastic process.

The mean field of a microscopic model can have several useful properties and can even be analysed with analytical methods. One method is the mean-field approximation, which allows approximation of the mean value of the (stochastic) mean-field by a closed, aggregated equation based-model - a so called mean-field model. Usually a ordinary differential equation [7] or difference equation [8] model results, but in case states of agents are continuous (as in our case) the method results in a partial-differential equation (PDE) model[9].

Key to find this mean-field model is to apply a so called mean-field theorem [10], a statement closely related to the famous Kolmogorow equations for Markov processes. If applied correctly, this theorem provides information how stochastic analysis of the single agents in the $\mathrm{AB}$ model results in an aggregated model for the mean-field.

\subsection{Application}

In order to apply the cited mean-field theorem, the stochastic processes of the single agents need to be analysed. This process is quite long and technical. Hence we refer to [9] to get an idea about how this can be done.

Let $F(a, t)$ and $M(a, t)$ denote the density of female and male $a$-year old individuals in Austria at time $t$, then the following model describes their dynamics:

\section{Corollary 2.1 (Mean-Field Model.)}

$$
\begin{gathered}
\frac{\partial M}{\partial t}-\frac{\partial M}{\partial a}=\alpha_{1}+\gamma_{1} \mathbb{1}_{[0, d t)}(a) \Psi(a, t)-M \delta_{1} \\
\frac{\partial F}{\partial t}-\frac{\partial F}{\partial a}=\alpha_{1}+\gamma_{2} \mathbb{1}_{[0, d t)}(a) \Psi(a, t)-F \delta_{2}
\end{gathered}
$$

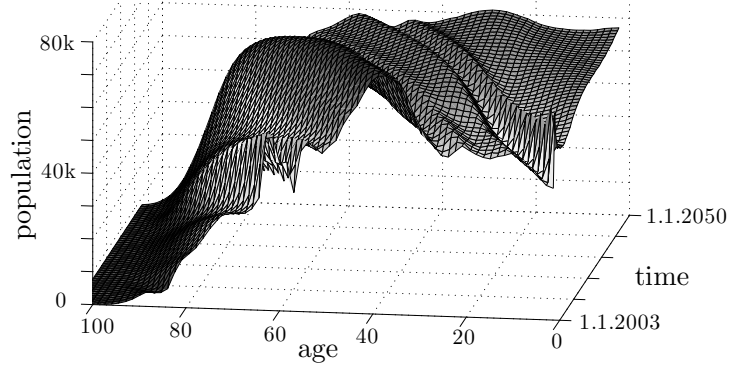

Figure 1: Simulation results for the PDE model. Density of male population for 47 simulated years.

with $\Psi(a, t):=\int_{\mathbb{R}^{+}} F(a, t) \beta(a, t) d a$. All $\alpha, \beta$ and $\gamma$ are functions of age and time and can directly be derived from [\#2]-[\#7]. The initial values are given by the initial density and the total population from [\#1] and [\#2]. Parameter dt poses for the AB model's time-step size.

The mean-field theorem guarantees asymptotic (with respect to the number of agents $N$ ) equivalence of the $\mathrm{PDE}$ solution and the $\mathrm{AB}$ population model. Hence the $\mathrm{AB}$ model qualitatively behaves according to some transport-equation.

Fig. 1 shows a surface-plot of the resulting density for male individuals from 2003-2050. The PDE model was simulated in MATLAB using a method-oflines scheme and its results almost perfectly match the results of the $A B$ model.

\section{Discussion}

While it takes almost half a day to simulate the $A B$ model the method of lines algorithm terminates after a few seconds. Hence it was possible to easily make quick scenario tests in order to verify the behaviour of the AB model. Also sensitivity analysis was supported: In case a parameter turned out to be very sensitive in the PDE model we paid close attention to it while analysing the $\mathrm{AB}$ model. Finally the $3 \mathrm{D}$ visualisation of the density in the PDE model inspired us to analyse diagonal cuts through the results of the $\mathrm{AB}$ model.

As the $\mathrm{AB}$ model is undoubted a much richer, applicable and flexible model, the PDE model is not intended to pose a substitute for it. Yet the time to derive, implement and execute the PDE model was still shorter than the duration of one single simulation run of the $A B$ model in Python, which made the mean-field model a useful tool to quickly gain first insights to support validation and verification in our case. 
Nevertheless it remains to be discussed: Is time invested into the derivation of this meta-model, not intended to be used directly, spent well?

\section{Acknowledgement}

K-Projekt DEXHELPP is supported by BMVIT, BMWFW and the state of Vienna via COMET - Competence Centers for Excellent Technologies. Programme COMET is processed by FFG.

\section{References}

[1] Andreassen L. Demographic Forecasting with a Dynamic Stochastic Microsimulation Model. 1993.

[2] Kelly S. Australia's Microsimulation Model DYNAMOD. 2003.

[3] Spielauer M. The LifePaths Microsimulation Model: An Overview. 2013.

[4] Malthus T. An Essay on the Principle of Population, as it Affects the Future Improvements of Society With Remarks on the Speculations of Mr. Godwin, M. Condorcet, and Other Writers. London. 1798.

[5] Bicher M, Glock B, Miksch F, Schneckenreither G, Popper N. Definition, Validation and Comparison of Two Population Models for Austria. In: Book of Abstracts, 4th International COnference on Business, Technology and Innovation 2015. Durres, Albania: UBT - Higher Education Institution. 2015; .

[6] Austria S. Demographisches Jahrbuch Österreich 2012. Verlag Österreich GmbH. 2012.

[7] Bicher M, Popper N. Agent-Based Derivation of the SIR - Differential Equations. In: Proceedings of the 8th EUROSIM Congress on Modelling and Simulation. Cardiff, Wales. 2013; pp. 306-311.

[8] Gast N, Gaujal B. A mean field approach for optimization in discrete time. Discrete Event Dynamic Systems. 2011;21(1):63-101.

[9] Deffuant G, Neau D, Amblard F, Weisbuch G. Mixing beliefs among interacting agents. Advances in Complex Systems. 2000;03(01n04):87-98.

[10] Tainaka Ki. Stationary pattern of vortices or strings in biological systems: Lattice version of the Lotka-Volterra model. Physical Review Letters. 1989; 63(24):2688-2691. 